\title{
Téoros
}

Revue de recherche en tourisme

\section{Des fous au service de la fête}

Table ronde animée par Jean Pelletier

\section{Jean Pelletier et Andrée Lamontagne}

Volume 5, numéro 2, juillet 1986

Tourisme, fêtes et événements

URI : https://id.erudit.org/iderudit/1080573ar

DOI : https://doi.org/10.7202/1080573ar

Aller au sommaire du numéro

Éditeur(s)

Université du Québec à Montréal

ISSN

0712-8657 (imprimé)

1923-2705 (numérique)

Découvrir la revue

Citer ce document

Pelletier, J. \& Lamontagne, A. (1986). Des fous au service de la fête : table ronde animée par Jean Pelletier. Téoros, 5(2), 12-17. https://doi.org/10.7202/1080573ar d'utilisation que vous pouvez consulter en ligne.

https://apropos.erudit.org/fr/usagers/politique-dutilisation/ 


\section{Des fous au service de la fête}

Table ronde animée par Jean Pelletier

Mise en forme du texte par Jean Pelletier assistée d'Andrée Lamontagne

Les responables de ce numero thematique ont souhaite connatire les points de vue d'organisateurs de festivals et d'évenements. Si selon les mois de Harvey Cox, chaque personne est un homo festivis, wous trouverez ci-dessous les propos et confidences de gens qui rravallent -souvent en riant-à faire chanter, danser, jouer et cellebrer les autres.

Une table ronde a permis de reunir six participants: la diversité des evénements et des personnages garantit une representarivite fort honorable. Avaient accepté de jouer le jeu Jean-Claude Arsenaul, directeur general du Marathon iniemational de Monireal, Frank Furtado, producteur delegue de I'International Bensons \& Hedges (concours intemational d'art pyrotechnique), Paul Dupont-Heber, directeur général du Festival d'été De Lanaudiere, Gilles Delorme, conseiller en gestion et excollaborateur de Quebec 1534-1984, Gil. les Déry, président du Camaval-Souvenir de Chicoutimi et Victor Simard, directeur des communications du sanctuaire de Sainte-Anne-de-Beaupre. Les opinions de Gilles Delorme soni reproduites dans un autre texte de ce numéro par l'auteur luiméme; celles de Victor Simard ont été regroupées dans un encadré pariculier comple tenu de la nature singulière d'un lieu de pelerinage.

L'organisation et l'animation de cente table ronde ainsi que le choix des extraits el leur mise en forme ont été assurés par Jean Pelletier, directeur général du Centre d'études du tourisme el André Lamontagne, érudiante au module de gestion et intenen. tion touristiques de I'UQAM.

Prenez connaissance de leurs expériences, ecouter les differences et trouvez les pers. pectives convergentes qui animent ces animareurs de ruches en fére au service de la fête.

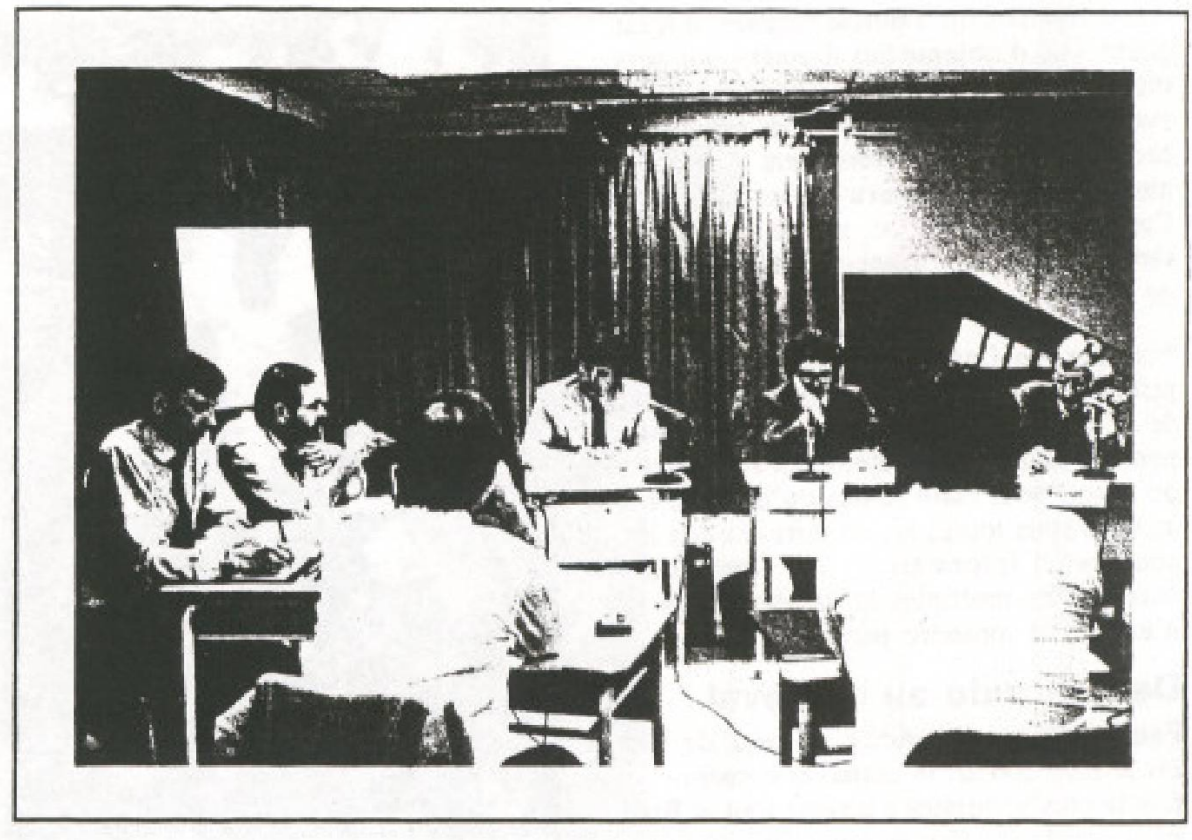

\section{L'aventure c'est l'aventure}

Sans trop de fausse modestie, les responsables des événements reconnaissent d'emblee que le talent et la chance ne sont pas les seules conditions du succes: les énergies, les efforts et les convicions sont des ingredienis de base necessaires.

Gilles Déry: La premiére clé du succès. c'est d'y croire vraiment et de prendre tous les moyens possibles et impossibles pour mener à bien le projet. C'est de travailler sur la spécificité de ce carnaval d'hiver â caractère historique qui permet de nous démarquer d'autres activités hivernales.

Jean-Claude Arsenault: Le secret de notre réussite: le travail. On a profité d'une vague qui était partie et on a réussi à placer l'organisation d'une façon telle que les gens maintenant disent qu'elle est très rodée. C'est difficile de dire des choses contre quelqu'un qui veut améliorer sa forme physique: on roule aussi là-dessus. C'est un des secrets de notre réussite. Le niveau international de l'événement est aussì un des eléments. On touche tout le monde.

Frank Furtado: Le secret du succès, c'est d'y croire. A l'origine des feux, personne $n^{\prime} y$ croyait, on était trois! On voulait ètre le plus gros, le plus beau naturellement.

Paul-Dupont Hebert: I faut y croire. Au départ, ça prend une idée folle ou un peu farfelue. Le plus souvent, c'est quand personne $n$ 'y croit que ça marche parce que le public veut avoir un projet original. On vend du rêve, du bonheur, de l'émotion, la santé, le loisir. Il faut réver un peu sinon, si c"est comme d'habitude, pourquoi le public embarquerait-il. Lorsqu'on veut ouvrir un nouveau volet, donner une nouvelle facette à quelque chose, les gens disent toujours " non. ça ne marchera pas'", Nous autres, on est sutr que ça va marcher parce que ça marche ailleurs. Dans Lanaudière, cela a demandé de la qualité et de la patience. C'est la neuvième saison cette anné, et ça a pris cinq, six ans avant que les gens en parlent et que les salles se remplissent. Avant les salles tiaient vides. 
Jean-Claude Arsenault: Il faut garder ça magique dans la tête des gens. Il faut amener des éléments nouveaux. On a toute une stratégie, continuellement repensée, pour communiquer à celui qui arrive au fil d'arrivé la magie de l'événement. Il y a un défi tous les jours et la joumée de l'événement c'est 150 dossiers qui doivent tomber à la seconde. C'est le danger d'avoir un événement de 24 heures ou de 9 heures qui a demandé 12 mois de préparation. Il ne faut pas gaffer sur un seul elément. On ne répète jamais le marathon de Montréal. On le recommence à chaque année. Remarquez que méme si des fois on se casse la gueule, ça vaut la peine parce que c'est le fun d'être en avant, de se dire: "les autres ne l'on pas encore fait". C'est une drogue quand tu touches à quelque chose qui est nouveau. C'est bien beau les claques dans le dos, les félicitations, les photos dans les journaux. A un moment donné, il faut aller plus loin en tant qu'individu.

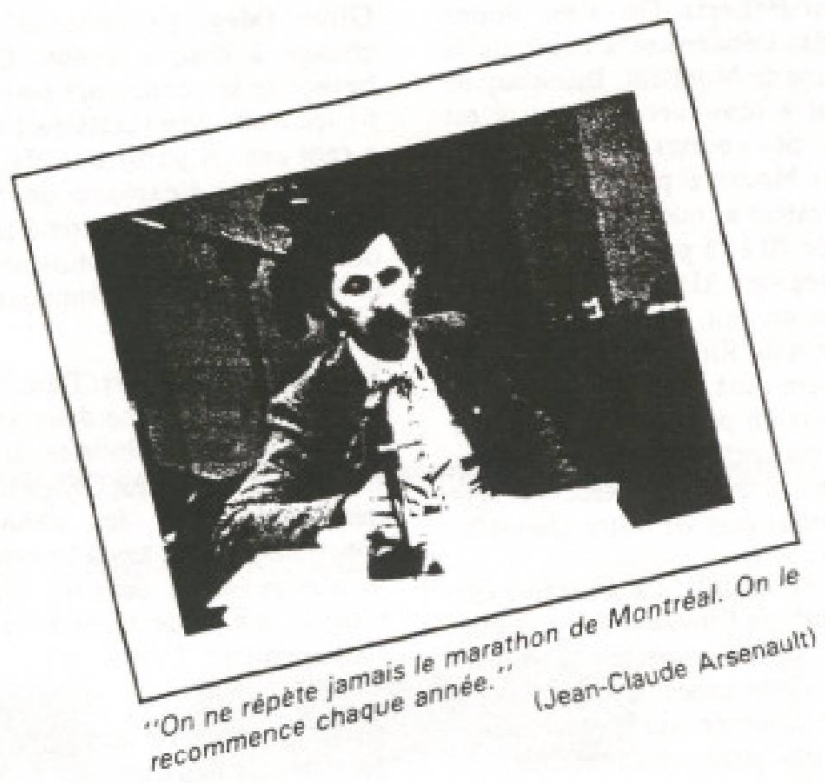

\section{Le tourisme en fête}

Les motivations de départ des organisateurs sont multiples tant au plan professionnel que personnel. Et souvent, ils en arrivent, parfois a leur grande surprise, à déplacer des foules qui viennent de partout el de très loin.

Gilles Déry: Je dirais qu'au moins $50 \%$ de la programmation est orientée vers le volet touristique. On a compris que c'était une dimension à exploiter si on voulait continuer à exister et à prospérer. Si on consulte les différents livres d'accueil que l'on garde à chaque année, on se rend compte que l'on déborde largement de la région du Saguenay-Lac-Saint-Jean au niveau touristique. On parle en termes de milliers de personnes venant de l'extérieur du Québec.

Frank Furtado: Personne ne s'attendait à un succès pareil l'année dernière. En termes touristiques cette année tous les hôtels m'appellent: c'est plein partout, ça se déplace de partout. C'est le plus beau spectacle au monde. Tout le monde veut $y$ venir. Je pense que cela attire énormément de monde, de la Nouvelle-Angleterre notamment.

Jean-Claude Arsenault: Notre événement a beaucoup de retombées touristiques parce que pour chaque marathonien, il y a un ou deux accompagnateurs. Donc, on parle de deux ou trois personnes qui mangent, dorment, louent une automobile, visitent le Québec et ainsi de suite. C'est incroyable, on touche vraiment le monde parce que Montréal est positionnée sur le méme pied que Paris, Londres, New-York. De plus, la télévision présente annuellement un produit

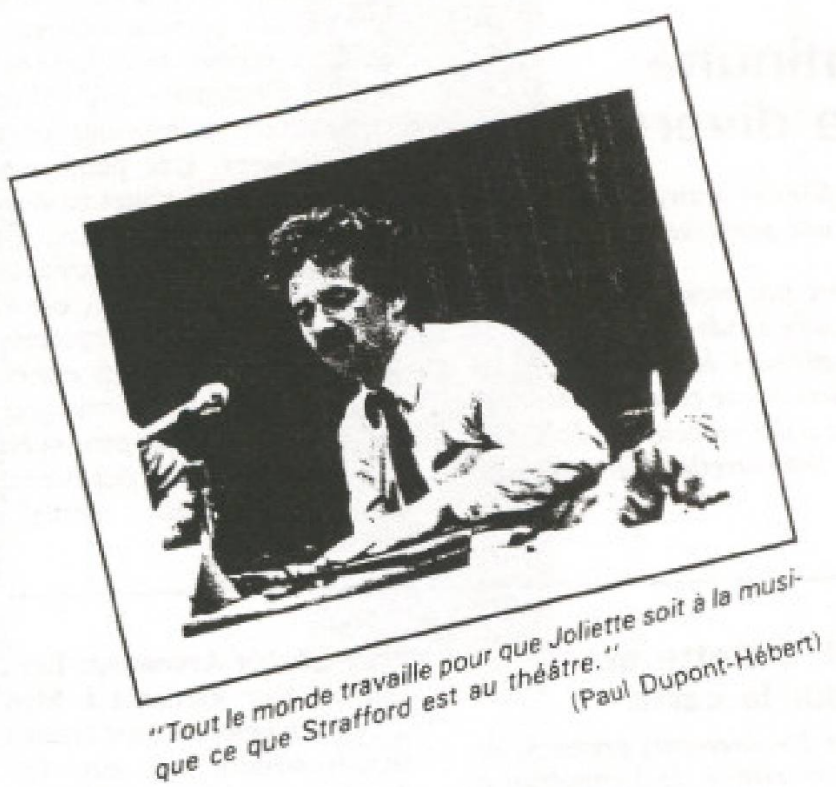

de haute qualité et de niveau international à travers le monde, exporte l'image du Québec er de Montréal.

Paul Dupont-Hébert: Notre dimension touristique, on la situe à trois niveaux. Au premier niveau, il y a les retombées directes en termes d'hébergement, de restauration, puisque 75 pour cent de notre public vient de l'extérieur de la région. Les amateurs de musique se déplacent car les festivals de musique classique sont rares. Le deuxième niveau, cest de faire connaitre une région à des gens qui ne la connaissent pas. Le troisieme niveau, c'est l'identification d'une région. Tranquillement, tout le monde travaille pour que Joliette soit à la musique ce que Strafford est au théátre.
Et ça, je pense que touristiquement, à très long terme, c"est intéressant parce que les gens vont s'apercevoir que c'est vraiment une région de musique, par le nom des rues, par toutes les terrasses et restaurants où des musiciens classiques sont engagés l'été, par des fanions musicaux posés sur tous les poteaux possibles. Joliette va s'appeler Joliette, ville de musique.

Gilles Déry: Le camaval, c'est sans doute l'activité historique la plus importante, du moins au Québec sinon au Canada, surtout durant la periode hivernale. On a une permanence en communication depuis maintenant deux ans. Son mandat, c'est d'aller chercher du monde à l'extérieur de notre territoire de réalisation. 
Paul Dupont-Hébert: On s'est donné comme mandat l'étalement à cause de la distance relative de Montréal. Beaucoup de gens viennent à plusieurs reprises: c'est toujours pour des courtes durées. On est de Montréal restent à coucher. Notre clientèle provient de 70 à 75 pour cent de l'extérieur de la région. Montréal, bien súr, Trois-Rivières un peu, on a beaucoup de monde qui vient de Rimouski, de Chicoutimi, de Rivière-du-Loup, de Québec et d'Ottawa. C'est un peu europten comme formule: on s"est aperçu que les. Européens qui étaient en vacances au Québec formaient 4 à 5 pour cent de notre clientèle.

Jean-Claude Arsenault: La personne qui vient à Montréal, on l'invite à voir une culture différente, une ville spéciale et un peuple particulier. Cette année, notre approche est totalement orientée sur l'Américain. Dans une de nos pages publicitaires, on peut voir l'Américain avec son sac de golf, ses "running shoes". On lui décrit tout ce qu'il va voir mais à Montréal, il va aussi découvrir des choses qu'il ne pourra jamais voir dans son pays. Et à un prix tellement abordable!

\section{La continuité dans la diversité}

Plusieurs des fertes et festivals prennent ou ont dejà pris une ampleur considerable.

Il $n$ 'en demeure pas moins nécessaire de tenter des coups de sonde sous la scène afin de mieux comprendre les exigences pour des organisations qui ne correspondent pas nécessairement à des madèles traditionnels d'entreprises industrielles ou commerciales. trop proche de Montréal pour que les gens

Gilles Déry: Le thème du carnaval est changé à chaque année. Cela implique beaucoup de recherches pour faire ressortir les traits dominants de l'histoire d'il y a cent ans. $\AA$ partir de cela, on voit si ça vaut la peine d'exploiter un thème. Cependant, je mettrais peut-être l'emphase sur la dimension programmation avant la gestion et la recherche de commanditaires.

Paul Dupont-Hébert: Dans le domaine du spectacle, on marche deux ans d'avance à peu pres. Nous, a Joliette, la programmation pour l'été 87 est tracée dans ses grandes lignes. Un des atouts quand tu fonctionnes deux ans à l'avance. c'est que tu $\mathrm{n}^{\text {'as }}$ personne dans les jambes lorsque c'est le temps de réserver des salles, de l'équipement.

Gilles Déry: Il y a une activité qui se prépare depuis deux ans qui va ettre formalisée probablement en 87 . Mais à part cette facette, le fonctionnement, le rouage, la mise en route de la "patente" annuelle se fait habituellement en septembre. Il est possible que l'on se réajuste et que l'on commence au mois d'aoüt, notamment au niveau des commanditaires. Remarquez qu'il y a toujours au départ une phase qu'on appelle d'orientation. C'est probablement le seul exercice théorique formel qui se fait annuellement. Une petite séance de planification où on remet en question le carnaval et ses orientations. Une fois par année, on redéfinit le carnaval, sa spécificité, son caractère. Et c'est à partir de là que l'on organise la programmation. C'est un perpétuel recommencement. Continuité au niveau de la thématique: le fait de demeurer historique pour survivre. Diversité par l'évolution des thèmes: il faudra toujours en ajouter d'autres!

\section{Chicoutimi, Joliette et Montréal sur la carte}

Les animaleurs d'événements prennent de plus en plus conscience de limportance tant siratégique qu'économique d'inviter et d'accueillir les visiteurs des autres pays. qu'ils soient participants ou vacanciers.

Frank Furtado: C'est réputé que Montréal est LE pays en ce moment. Les États-Unis -plusieurs villes américaines- se demandent comment il se fait qu'ils n'ont pas pensé aux feux avant nous. Notre festival est le plus long, le plus gros, c'est vraiment dans l'esprit d'un concours international. Les pays sont en réalité des firmes qui participent. Il y a une immense compétition dans le domaine des feux d'artifice à travers le monde. Je pense que le succès des feux dépend vraiment de cet aspect concours entre les pays.

Jean-Claude Arsenault: Les 20, 25 coureurs d'élite viennent à Montréal parce qu'on a développé autre chose qu'une relation monétaire avec eux. On a toujours donné la priorité au volet populaire. Beaucoup de gens, surtout les Américains, veulent essayer d'autres marathons que les leurs. Les inscriptions des Américains ont augmenté de 150 pour cent: la valeur du dollar et l'image d'une ville gastronomique $y$ font pour beaucoup.

Gilles Déry: La dimension internationale, il faudra y penser encore mieux éventuellement.

Paul Dupont-Hébert: Cette année, on a fait une conférence de presse à Paris justement pour sensibiliser les Français, qui vont venir au Québec cet été, au fait qu'il existe un festival de musique qui est à cóté

\section{Les commanditaires publics et privés}

Le système nerveux de la fêre commande evidemment des conditions facilitantes au niveau du financement. Entre les attentes des institurions publiques (et politiques) et les exigences parfois contraignantes des "sponsors", il faut parfois savoir - ou apprendre! - à naviguer à vue.

Gilles Dery: Le financement, c'est toujours un problème important à chaque année. Tu fais un budget, tu te dis que ça prend tant de revenus qui vont venir de différentes sources, soit des activités elles-mémes, soit d'une campagne de levée de fonds, soit de subventions gouvernementales. II n'y a rien d'acquis.

Jean-Claude Arsenault: Le marathon est financé à 18 pour cent par lé gouvernement du Québec. Une aide gouvernementale de l'ordre de 20 pour cent est une proportion valable. Le degré auquel les gens prennent à coeur le succès de leur événement et les responsabilités qui s'y rattachent est directement relié au pourcentage d'énergie que vous mettez dans la recherche de fonds. Avec un financement public de l'ordre de 100 pour cent, on a vu à un moment donné qu'il y avait un certain désintéressement. D'ailleurs, plus la portion du gouvernement est grosse, plus les réunions, les contrôles, les groupes de travail et tout cé qui s'en suit augmentent.

Frank Furtado: Dans les organisations, quand il y a un gouvernement impliqué fédéral, provincial, municipal - c'est presque toujours un problème. Il y a toujours quelqu'un qui veut tirer son épingle du jeu. Pis, il y a toujours trois personnes au lieu d'une! Dans la minute oú il y a un gouvernement impliqué, ça coúte plus cher: il y a trois personne pour faire la job d'une. de Montréal et qui dure tout l'été.
Paul Dupont-Hébert: À chaque année, il faut se retrousser les manches, surtout avec un changement de gouvernement. Il faut ramer un peu plus même si nous sommes députés, de bons ministres. Durant l'été, on profite des programmes Canada au Travail, Défi, programmes de création d'emplois. Pour ce qui est des commandi= taires, ça va. Nous. ce qui nous sauve, c'est la billetterie. Le défi, c'est surtout de vendre suffisamment de billets pour arriver.

Frank Furtado: Tout le monde en profite dans un sens. Les feux sont un projet de La Ronde. L'AMARC est une organisation para-municipale. La ville de Montréal retire des taxes, entre autres.

Gilles Déry: Actuellement, sur un budget de 9000005 . c'est un ratio un tiers-deux tiers que nous avons. C'est-à-dire un tiers du gouvernement (municipal, provincial et très bien placés parce que l'on a de bons 


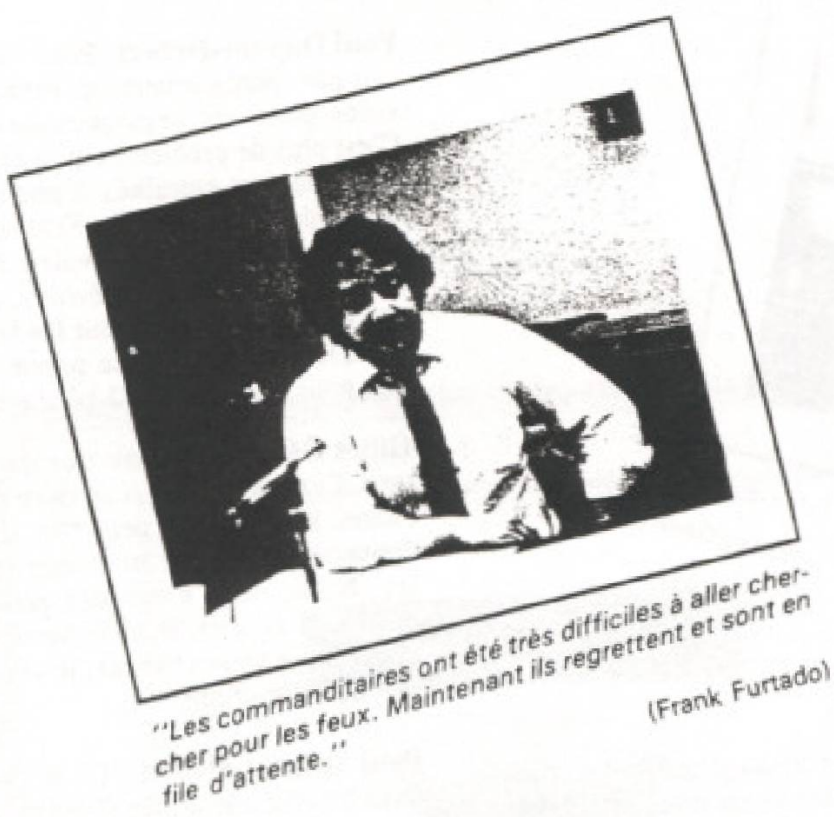

fédéral) et deux tiers d'autres sources, soit des activités de financement, soit des commandites. On se rend compte qu'il faut s'en aller vers des commandites de plus en plus. On n'a pas le choix.

Frank Furtado: Il faut que ce soit une organisation comme l'AMARC qui chapeaute les feux parce que financièrement c'est très dispendieux même si nous avons de gros commanditaires. Au départ, le conseil d'administration de l'AMARC a investi une bonne somme d'argent en disant "on va prendre le risque" " L'AMARC a vrajment sauté à pieds joints. Les commanditaires ont été très difficiles à aller chercher pour les feux. Maintenant, ils regrettent et sont en file d'attente.

Jean-Claude Arsenault: Le succès des événements ressemble beaucoup à ceux de l'entreprise privée. Ta réussite est basée sur ce que tu mets en avant.

Gilles Derry: On a toujours besoin de se battre pour avoir des subventions ou quoi que ce soit parce que plusieurs s'imaginent qu'on ne peut pas déplacer les gens en hiver.

Paul Dupont-Hébert: Nous, on s"est aperçu que pour faire un événement, pour le vendre aux commanditaires, pour donner de l'importance, ça prenait des artistes de plusieurs pays. Donc, on a toujours au minimum une douzaine de pays quil sont représentés.

Jean-Claude Arsenault: Pour vendre un événement à des commanditaires, le plus gros attrait c'est la couverture media au plan international. Si vous faites venir les meilleurs au monde, on va vous certifier que ça va être télévisé. Alors. on invite 20. 25 pays pour avoir une compétition avec un gros $C$ en sachant que derrière, il y a 10000 coureurs: c'est aussi un gros $\mathrm{C}$ mais pour des raisons personnelles. Pour pouvoir se positionner au méme niveau que New-York, Boston, Paris, le Marathon de Montréal a joué la carte du spectacle. Le spectacle, ce sont les 35 antilopes en avant qui roulent à $2 \mathrm{~h} 10,2 \mathrm{~h} 11$ pour le marathon. On peut se reconnaittre en eux en disant que c'est la quintessence de la course à pied. Cela fait un spectacle télévisé de très haute cote et ça plait fortement aux coureurs ordinaires de voir que, devant eux, les idoles sont là. Le niveau international est très important pour la télévision, mais la participation populaire au niveau des Québécois, c'est ça qui est important pour les commanditaires. C'est aussi important pour la télévision.

Gilles Déry: Des fois, un seul commanditaire ça crée des contraintes. On devient très dépendant. Il faut trouver un bon " rage" de commanditaires qui n'entreront pas en conflit, bien sûr.

Frank Furtado: Nous, ça porte le nom du commanditaire qui est appuyé par deux medias, qui sont La Presse et $C K A C$. C'est 1'Intemational Bensons \& Hedges présente par La Presse et CKAC. Dans cet esprit là. un commanditaire unique, c'est avantageux pour eux. Avant, Bensons \& Hedges n'était pas vraiment mis en évidence dans un événement, ils cherchaient. Quand ça porte le nom du commanditaire, c'est fantastique.

Paul Dupont-Hébert: C'est une réaction en chaine. Ça devient presque une mode. Tout le monde veut avoir son événement sensationnel. Tout le monde veut ètre en avant, la locomotive.

Jean-Claude Arsenault: On fait affaire avec des gros commanditaires mais il y en a très peu. Parce que l'événement a des retombées surtout nationales et internationales, il attire des commanditaires de niveaux national et international.
Paul Dupont-Hébert: Je trouve ça très facile de travailler avec les commanditaires. C'est clair ce qu'ils veulent, on négocie, on s'entend sur une affaire et ça roule. Ce sont des gens qui sont là pour la visibilité. C'est clair, net et précis. Ça se met sur papier. Ils visitent les lieux. Ils sont méme souvent généreux au niveau de leur implication. ils font plus que ce qui est convenu. Ça roule très bien. Avec eux, on essaie d'améliorer de plus en plus les rapports. Cene année, il y a quelqu'un au bureau pour les accueillir, etc.

Jean-Claude Arsenault: Après un certain nombre d'années de réussite, on va $s$ appuyer uniquement sur la participation des sources privées. Il ne faut pas oublier que la situation économique se reflète très rapidement sur les commanditaires qui peuvent vouloir changer leur stratégie de promotion et de publicité.

Gilles Déry: II y a une maturation qui s'est faite autant de la part des organisateurs que de la part des commanditaires qui sont très ouverts à s'associer à une formule gagnante. Ca nous force à justifier le bienfondé d'un investissement dans notre carnaval. Ils le comprennent bien.

Paul Dupont-Hébert: En général, les gouvernements vont aller de plus en plus vers des formules complémentaires du genre "un dollar public pour un dollar privé".

\section{Une affaire d'organisation}

Entre les contraintes budgétaires et l'apport des intéressés bien intentionnés. les responsables des fetes doivent faire face à la musique. Les aléas possibles des structures légères peuvent-ils être facilement contrôlés?

Gilles Déry: L'équipe de permanents du carnaval est de six personnes. A cela, il faut ajouter une filiale qui travaille environ six mois par année avec six autres personnes. La filiale, Les Industries du Camaval, fabrique les costumes d'époque, etc. On essaie le plus possible de décentraliser la structure sauf qu'évidemment, il $y$ a des gens qui vont prendre des décisions. On a une structure qui est faite pour prioriser trois grands secteurs: finances, communications, activités. Il y a des vice-présidents responsables de ces trois secteurs, avec des permanents qui leur sont attachés. Viennent par la suite, des directeurs qui ont des coordonnateurs pour les différentes facettes. Et c'est au coordonnateur finalement de s'assurer qu'il y aura des présidents pour les différents comités, les différentes activités, qui fera le suivi là-dessus et qui aura des comptes à rendre au conseil d'administration sur une base régulière. Tu peux faire ressortir certains points communs, certains 


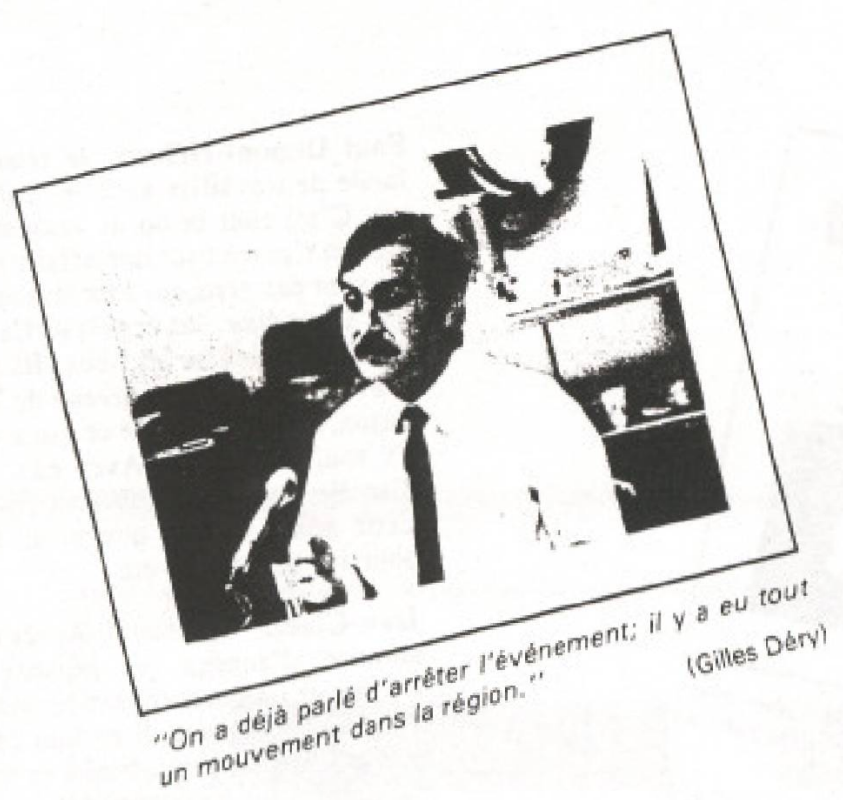

traits principaux mais il faut vraiment s'ajuster à chacune des activités, à chacune des particularités, aux gens avec qui tu travailles, aux sources de financement, aux relations politiques, etc. De telle sorte que c'est assez difficile. C'est malheureux en fait, parce que souvent il y a du temps perdu. Mais finalement, à l'usage le temps arrange bien les choses.

Jean-Claude Arsenault: Si on parle de communication interne entre les exécuteurs, on peut perdre du temps lorsqu'il y a trop de communication sur le comment on devrait faire. Pour des organisateurs d'événements qui ont des échéanciers critiques, il est très important, pour la qualité de l'événement, de compter sur la vitesse d'exécution. Que les choses tombent à la seconde!

Paul Dupont-Hébert: Voici une autre expérience vécue chez nous. A un momen donné, le président a pris l'initiative de dire que si on ne risquait pas d'engager un permanent, on n'aurait pas de commanditaire. Cependant, ce que j'ai préconisé, c'était le moins possible de permanents, le plus possible de pigistes et le moins possible de bénévoles. Si tu engages quelqu"un pour un mois, il travaille pendant un mois, il connaitt son affaire. Son expertise, il l'a. Il en a fait. Il connaît ça. Au bout d'un mois. c'est fini et il sait que. s'il n'est pas bon. il n'aura pas de contrat l'année prochaine.

Gilles Déry: Il faut aussi planifier nos activités en concertation, c'est un peu ce que I'on fait. Par exemple, avec Québec, i] y a plusieurs années, les deux camavals avaient lieu en même temps. On s'est rendu compte que c'était fou parce qu'on touchait quand même une clientele qui était en partie la même dans les deux cas, des gens qui aiment des activités hivernales. Alors, on s'est mis d'accord à un moment donné pour se compléter au niveau du calendrier. De telle sorte que le Carnaval de Québec commence une semaine avant nous, mais nous on termine une semaine plus tard. Finalement, on se rend compte qu'il y a des touristes qui se promènent entre les deux. C'était rejoindre l'utile à l'agréable. De toute façon, on n'avait pas à se compétitionner l'un l'autre mais plutót à travailler ensemble. Et ça c'est heureux.

\section{De l'utilité des bénévoles}

Les citoven-nes qui ont le goüt de donner volontairement temps et argent representent des banques de ressources qu' $u$ faur savoir exploiter el sarisfaire. C'est un defi qui $n$ 'est pas nécessairement facile.

Jean-Claude Arsenault: S'il y a quelque chose dont nous sommes fiers, c' est que le marathon appartient aux 3000 bénévoles. Vous seriez surpris de voir combien de groupes ou de familles monopolisent des points d'eau, des points d'éponges depuis ? ans.

Paul Dupont-Hébert: Pour ma part, je ne suis pas spontanément très preneur de benévoles dans les organisations artistiques. C'est plus de problemes qu'autre chose tant qu'ils ne sont entraînes. Cette arinée on va développer les Amis du Fesrival. On aura ainsi une armée de bénévoles. Mais quand tu as une armée de bénévoles, ça te prend une armée de monde pour les faire travail. ler. Dans le fond, ça te prend 10 personnes pour encadrer 100 bénévoles.

Gilles Déry: Je conçois que dans la structure d'une activité qui se déroule sur plusieurs mois, c'est peut-être difficile de conserver un intérêt au niveau des bénévoles. Nous, nous avons des gens qui nous disent "l"an prochain, je veux faire telle chose ou je veux changer, je veux m 'occuper de telle chose"

Paul Dupont-Hébert: Ça a un très bon fond d'avoir une armée structurée de bénévoles. Mais comme la táche est grande, j'ai choisi d'engager un ou deux pigistes pour un ou deux mois chacun et d'être sûr que tout arrive a la bonne place, au bon moment. Je suis plus enclin à faire contribuer les bénévoles à des activités qui donneront de l'argent à l'organisation plutồt que de les mettre dans l organisation ellemème. Les gens qui vont vouloir mettre du temps, on va leur confier d'organiser des activités à l'intérieur et à l'extérieur du festival. C'est-à-dire que si on organise un souper-bénéfice et qu'ils veulent en orga= niser un deuxième, ils l'organiseront. Les Amis du Festival, comme les Amis du Musée des Beaux-Arts, devront payer une cotisation. Il y aura différentes catégories d'amis selon le montant payé. Ca c'est pour aller chercher ceux qui veulent contribuer au festival et qui ne savent pas comment. Ils seront employés pour des services d'accompagnement, pour la vente de sou= venirs et pour la sécurité de certaines soirées. On va tranquillement leur faire faire un travail valorisant.

\section{Les ramifications de la fête}

Les fetes et festivals font flèche de tout bois. Certaines retombees, maintenant connues, $n$ 'avaient pas été prévues, woire même imaginées au poin de dépar. Comme quoi, les parametres coûts-bénéfices permettent habinellemen de mieux mesurer l'ensemble des impacts d'un evénement.

Jean-Claude Arsenault: Le marathon est devenu un des objets de la fierté des Montréalais. Qui plus est, une demande presqu'instantanée de courses de plus courte distance s"est exprimée pour pouvoir s'entrainer pour le marathon: demande qui a provoqué une vague incroyable de courses à pied et la découverte de nouveaux organisateurs.

Gilles Déry: On a dêjà parlé d'arrêter l'évênement. Il y a eu tout un mouvement dans la région. On s"est rendu compte que c'était plus important qu'on ne le pensait que cétait difficile à remplacer en plein hiver. Le carnaval permet aux plus vieux de se retrouver et de recréer des choses qu'ils ont vécues. Il permet aussi aux jeunes de prendre contact avec l'histoire d'une façon un petit peu plus concrète que dans les bouquins. Et on doit reconnaitre que la 
participation à ce niveau-là est très forte. Après l'impact économique, c'est la fierté des populations qu'il est intéressant d'observer.

Frank Furtado: Les feux sont rapidement devenus une locomotive. L'International est un des premiers d'une série de festivals qui s'échelonnent tout au long de l'été̉ montréalais. Les feux ouvrent la saison touristique québécoise en faisant la une des journaux pendant plus d'un mois. Ce qu'il $y$ a de fantastique, $c$ 'est que c'est un festival qui se tient à l'extérieur, beau temps, mauvais emps. Ajoutons qu'un njveau de I'importation, les douanes disent assister à une augmentation de 400 pour cent en ce qui concerne le matériel pyrotechnique. Que dire de la qualité des spectacles aussi: les feux pyro-musicaux n'existaient pas ici avant l'expérience réussie de 1985.

\section{Des projets d'avenir}

Nos fous merveilleux au service de la fête osent a peine parler de certains de leurs projets. Prenez connaissance de quelquesunes de leurs intentions et surveillez leurs acrions.

Jean-Claude Arsenault; Il va y avoir un grand prix de cyclisme à Montréal avec Bernard Hineault et les autres. Ils vont tous être ici. A Montréal, il est temps que l'on ait un événement cycliste de niveau international.

Paul Dupont-Hébert: A moyen terme, le Festival veut créer régulièrement des productions que nous ferons tourner à travers le Canada comme ambassadeurs de l'événement.

Jean-Claude Arsenault: Nous avons déjă imaginé décorer le pont Jacques Cartier qui serait illuminé en fleurs de lys. On a tous les plans. On aurait une chute d'eau qui partirait du pont avec un rayon laser pour le soir. On fermerait la ville de Montréal avec 10 arches ethniques où chacun des groupes de Montréal participeraient. J'en réve à chaque fois que je vois des gens qui réalisent des choses.

Paul Dupont-Hébert: Nous, à Joliette. voudrions que tous les grands artistes qui viennent dans Lanaudière donnent des cours l'après-midi à des gens de la même discipline. On organiserait des cours de mailtres. On a de grandes vedettes qui arrivent et qui repartent le lendemain; on aimerait les retenir pour qu'elles puissent témoigner et leur faire faire un peu de.. tourisme.

\section{0 \\ pèlerins-voyageurs chaque année sur la Côte-de-Beaupré}

Sainte-Anne de Beaupré, lieu de pèlerinage, de tourisme religieux... mais il s'agit aussi d'une ferte, celle de Sainte-Anne, le 26 juillet... et toute l'anné durant. Beaucoup de ressemblances avec les autres fetes et événements mais plusieurs différences: les morivations des visiteurs sont doubles, spirituelles et touristiques, l'organisation est etale dans le temps, il $n$ 'y a pas de subvention.

Les opinions de Victor Simard, directeur des communications du sanctuaire, sont ici regroupees.

Sainte-Anne de Beaupré, sans oublier tout ce qui s'y rattache, $n$ "est pas un simple lieu que l'on visite comme on le fait pour d'autres endroits historiques. C'est un lieu artistique et un lieu universel.

De tout temps, il y a eu des milliers de pelerins qui ont quitté leur demeure pour aller à la rencontre de leurs dieux. Etre pelerin, c'est briser la routine, partir, affronter les dangers de la route, se dépouiller devant la présence divine, pouvoir enfin regarder, toucher, redevenir un homme nouveau. Le pèlerinage consiste, dans son essence, à aller dans un lieu à la rencontre de l'autre et à sortir de la vie quotidienne, de ses habitudes, sortir presque de soi pour se mettre en situation de rencontrer l'autre. Dans I'histoire de toutes les grandes religions, les pèlerinages sont liés aux origines ou à des faits importants de l'histoire.

Sainte-Anne de Beaupré, c'est la foi d'un peuple. C'est pour ça que les gens $s^{\prime} y$ retrouvent et y viennent. Il n'y a pas un foyer (ou presque) où au moins un membre n'a pas fait une visite à Sainte-Anne: on appelle ça du tourisme religieux.

On est situé a $25 \mathrm{~km}$ de la Capitale et à $300 \mathrm{~km}$ de la métropole. Pour venir chez nous, il faut que les pèlerins traversent la province. Il faut qu'ils dorment, mangent, séjoument. Ils découvrent des choses, ils font un circuit de tourisme religieux en passant par l'Oratoire à Montréal, le Cap-dela-Madeleine en Mauricie. Ils font exacte-

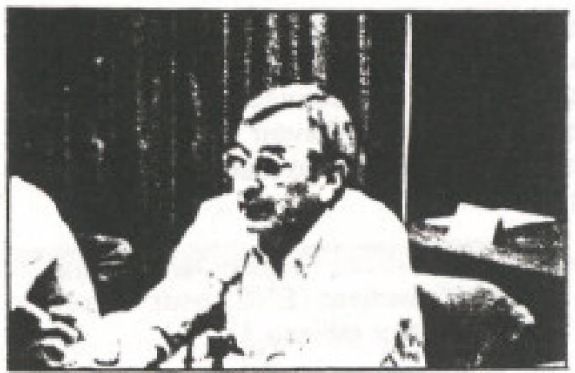

Victor Simard: "Sainte-Anne de Beaupré, ca fait vive une region."

ment ce qu'ils feraient à Paris: ils visiteraient Notre-Dame (la motivation sera la. sans qu'elle soit affichée).

1200000 personnes qui se déplacent, ça a des retombées économiques. Il faut des infrastructures: nous, ça nous regarde pas même si nous pouvons toujours les encourager.

Une des caractéristiques du sanctuaire de Sainte-Anne de Beaupré, ce sont les gens qui y viennent, s'organisant entre eux. Nous sommes là pour les recevoir. On fait enormément attention à l'accueil personnel.

On a comptabilisé 12250 heures en bénévoles pour l'accueil des malades. Toutefois. il faut une structure pour les encadrer. I faut qu'ils soient à leur place. Ca prend de la patience. Mais une fois que le train est parti, on a beaucoup à recevoir d'eux. $\mathrm{Ca}$ fait une infrastructure extrèmement forte. Par définition, un bénévole c'est le type qui n'est pas payé mais qui paie beaucoup pour travailler.

À Sainte-Anne, on n'a pas de problème de financement. Nous ne recevons aucune subvention. Il ne faut pas oublier qu'il y a une communauté religieuse en arrière et nous ne sommes pas des salariés. Tout ce qui entre est réinvesti.

Sainte-Anne de Beaupré ça fait vivre une région. Je n'ai pas les outils pour évaluer les impacts mais ça se développe toujours.

\section{Deux mots de la fin}

Les responsables des événements invitent le secteur privé comme les gouvernements à se complêter pour en arriver à des actions mieux planifiés.

Frank Furtado: Du point de vue touristique, il y a lieu qu'éventuellement certains intervenants, assurent un leadership pour garantir une meilleure concertation.
Gilles Déry: Si l'on veut développer le domaine touristique au Québec, il faudra en avoir vraiment une préoccupation cons. tante et des gens qui ne vont penser qu"à ça, notamment au plan de la responsabilite ministérielle. $f$ 